DESY 09-168

\title{
Possible cosmogenic neutrino constraints on Planck-scale Lorentz violation
}

\author{
David M. Mattingly ${ }^{1}$, Luca Maccione ${ }^{2}$, Matteo Galaverni ${ }^{3}$, \\ Stefano Liberati ${ }^{4,5}$, Günter Sigl $^{6}$ \\ 1 University of New Hampshire, Durham, NH 03824 \\ 2 DESY, Theory Group, Notkestraße 85, D-22607 Hamburg, Germany \\ 3 INAF-IASF Bologna, Via Gobetti 101, I-40129 Bologna, Italy \\ 4 SISSA, Via Beirut, 2-4, I-34151, Trieste, Italy \\ ${ }^{5}$ INFN, Sezione di Trieste, Via Valerio, 2, I-34127, Trieste, Italy \\ ${ }^{6}$ II. Institut für Theoretische Physik, Universität Hamburg, Luruper Chaussee 149, \\ D-22761 Hamburg, Germany \\ E-mail: davidmmattingly@comcast.net, luca.maccione@desy.de, \\ galaverni@iasf .bo.it, liberati@sissa.it, guenter.sigl@desy.de
}

\begin{abstract}
We study, within an effective field theory framework, $O\left(E^{2} / M_{\mathrm{Pl}}^{2}\right)$ Planckscale suppressed Lorentz invariance violation (LV) effects in the neutrino sector, whose size we parameterize by a dimensionless parameter $\eta_{\nu}$. We find deviations from predictions of Lorentz invariant physics in the cosmogenic neutrino spectrum. For positive $O(1)$ coefficients no neutrino will survive above $10^{19} \mathrm{eV}$. The existence of this cutoff generates a bump in the neutrino spectrum at energies of $10^{17} \mathrm{eV}$. Although at present no constraint can be cast, as current experiments do not have enough sensitivity to detect ultra-high-energy neutrinos, we show that experiments in construction or being planned have the potential to cast limits as strong as $\eta_{\nu} \lesssim 10^{-4}$ on the neutrino LV parameter, depending on how LV is distributed among neutrino mass states. Constraints on $\eta_{\nu}<0$ can in principle be obtained with this strategy, but they require a more detailed modeling of how LV affects the neutrino sector.
\end{abstract}

\section{Introduction}

Over the last fifteen years there has been consistent theoretical interest in possible small deviations from the exact local Lorentz Invariance (LI) of general relativity as well as a flourishing of observational tests. The theoretical interest is driven primarily by ideas in the Quantum Gravity (QG) community that Lorentz invariance may not be an exact local symmetry of the vacuum. The possibility of outright Lorentz symmetry violation $(\mathrm{LV})$ or a different realization of the symmetry than in special relativity has arisen in string theory [1, 2], Loop QG [3, 4, 5], non-commutative geometry [6, 7, 8, 9], spacetime foam [10], some brane-world backgrounds [11, and condensed matter analogues of "emergent gravity" [12]. As well, allowing the fundamental theory of gravity to be non-relativistic in the ultraviolet can make gravity renormalizable while avoiding some 
of the other pathologies that plague renormalizable gravitational actions with higher derivative terms [13].

Constructing useful tests for the various active models and ideas is therefore vital. Since there are so many theoretical models around, a good approach is to work within a calculable framework where all possible LV terms are parameterized. Each theory then picks out a certain combination of terms which can be constrained. In this vein, a standard method is to simply analyze a Lagrangian containing the standard model fields and all LV operators of interest that can be constructed by coupling the standard model fields to new LV tensor fields that have non-zero vacuum expectation valuest.

All renormalizable LV operators that can be added to the standard model in this way are known as the Standard Model Extension (SME) [16]. These operators all have mass dimension three or four and can be further classified by their behavior under CPT. Higher mass dimension operators can be systematically explored as well, which is useful in case the naive EFT hierarchy breaks down due to other new physics (e.g. SUSY) or quantum gravity introducing a custodial mechanism for the renormalizable operators in the infrared.

The CPT odd dimension five kinetic terms for QED coupled to a non-zero vector field were written down in [17] while the full set of dimension five operators with a vector were analyzed in [18]. The dimension five and six CPT even kinetic terms for QED for particles coupled to a non-zero background vector, which we are primarily interested in here, were partially analyzed in [19]. The full set of dimension five and six operators for QED has recently been introduced in [20]. It is notable that SUSY forbids renormalizable operators for matter coupled to non-zero vectors [21] but permits certain nonrenormalizable operators at mass dimension five and six.

Many of the parameterized LV operators have been very tightly constrained via direct observations (see [22, 23, 14, 24] for reviews). In particular, the dimension five and six CPT even operators for $\mathrm{LV}$ with a vector field have been recently directly $\$$ constrained in the hadronic sector by exploiting ultra-high energy cosmicray observations [26] performed by the Pierre Auger Observatory (PAO). Indeed, the construction and successful operation of this instrument has brought UHECRs to the interest of a wide community of scientists and it is expected to allow, in the near future, the assessment of several problems of UHECR physics and also to test fundamental physics (in particular Lorentz invariance in the QED sector) with unprecedented

$\ddagger$ There are other approaches to either violate or modify Lorentz invariance, that do not necessarily yield a low energy EFT (see [14] and refs therein). However, these models do not easily lend themselves to particle physics constraints as the dynamics of particles is less well understood and hence we do not consider them here. In particular, we remark here that ideas of deformation, rather than breaking, of the Lorentz symmetry (see, e.g., [15]) do not have an ordinary-EFT formulation, hence they cannot be tested with the arguments presented in this work.

$\S$ Notice that all these operators can be indirectly constrained by EFT arguments [25], as higher dimension LV operators induce large renormalizable ones if we assume no other relevant physics enters between the $\mathrm{TeV}$ and $M_{\mathrm{Pl}}$ energies. SUSY, however, is an example of new relevant physics that can change this. 
precision [27, 28, 29].

The UHECR constraints [30, 31, 32, 33, 34, 35, 26, 36] rely on the behavior of particle reaction thresholds with LV, which are one of the best methods in the EFT approach to constrain nonrenormalizable LV operators. Many LV operators give modified dispersion relations for free particles, where the energy as a function of momentum deviates slightly from the special relativistic form. For threshold reactions what matters is not the size of the LV correction to the energy compared to the absolute energy of the particle, but instead the size of the LV correction to the mass of the particles in the reaction. Hence the LV terms usually become important when their size becomes comparable to the mass of the heaviest particle. If the LV term scales with energy as $E^{n}$, then this critical energy is $E_{c r} \sim\left(m^{2} M_{\mathrm{Pl}}^{n-2}\right)^{1 / n}$ [37]. According to this reasoning, the larger the particle mass the higher is the energy at which threshold LV effects come into play. This is why $\gtrsim \mathrm{TeV}$ electrons and positrons, but not protons, can be used to constrain $n=3 \mathrm{LV}$ [38], and why UHE protons are needed to obtain constraints on hadronic LV with $n=4$ scaling (which corresponds to CPT even mass dimension five and six operators).

From this point of view, neutrinos, with their tiny mass of order $m_{\nu} \simeq 0.01 \mathrm{eV}$ [39], are in principle the most suited particles to provide strong constraints on LV, at least for reactions involving only neutrinos. One such reaction is that of neutrino oscillation. Indeed, for a decade neutrino oscillations have proven to be excellent tests of the SME and other LV models [40, 20, 41, 42, 43] as when the LV corrections are near the neutrino mass, the oscillation pattern can change as a function of energy, direction and mass. ICECUBE may even be able to probe dimension six operators with time of flight techniques with TeV neutrinos from distant Gamma Ray Bursts [44. UHECR experiments have also the capability of placing constraints on SME parameters by exploiting neutrino oscillations [45]|W. One can construct complementary and in some measures even more sensitive neutrino tests of higher dimension operators by leveraging observables which deviate more strongly from their special relativistic values as the neutrino travel distance increases. One such observable is found to be related to UHE neutrino spectrum observations.

Despite the threshold being low for LV effects to kick in, neutrinos with ultra-high energy are necessary to achieve a signal, as they interact so weakly that the phase space for a LV reaction must be huge to generate an appreciable rate. This requirement implies that, since the LV terms and hence the phase space grow with energy, very large energies are needed. Indeed, for renormalizable operators, where the phase space does not grow quickly enough, reactions of the type we consider here never achieve the necessary rate given current bounds on their coefficients, even for UHE neutrinos over cosmological distances [46, 40]. However, for the CPT even non-renormalizable operators the situation is different, and we find that for energies of order $10^{17} \mathrm{eV}$ there can be a significant modification of the spectrum. Next generation neutrino detectors

\| We notice that in the same work [45] the process of neutrino decay is discussed, but in a different context than what we consider here. 
such as ANITA [47] and SuperEUSO [48, 49] are sensitive to neutrinos of energies $>10^{19} \mathrm{eV}$. Further experiments, like the planned ARIANNA [50, 51] and IceRay [52], will cover the range $10^{17} \div 10^{20} \mathrm{eV}$.

In the present work we study how limits on the absolute scale of non-renormalizable CPT even LV neutrino parameters can be obtained from UHE neutrino observations. In particular we will determine the energy scale where the neutrino spectrum might begin to deviate as a function of the size of the LV coefficients. With three neutrino species as well as assorted light leptons possibly involved, the spectrum as a function of various LV parameters can only be computed by a detailed parameterized numerical search. At this stage, where LV in this sector is only speculative, we feel that such a search is unwarranted. However, we will show the "best case" scenario for a LV neutrino signal with dimension six operators as well as a scenario where a signal in the UHE spectrum is much more subtle even though LV is still relatively strong.

This paper is structured as follows. In section 2 we describe the theoretical LV framework in which we will derive the LV effects on the UHE neutrino spectrum. In sections 3 and 4 we will give general information on the standard understanding of UHE neutrino generation by UHECRs, and we will present and detail the main LV reactions possibly affecting their spectrum. Section 5 is devoted to present results in our test cases. In Section 6 we discuss the possible role of other processes than neutrino splitting. In section 7 we will report our final remarks and conclusions.

\section{Theoretical framework}

In order to study the phenomenological consequences of LV induced by QG, the existence of a dynamical framework in which to compute reaction thresholds and rates is essential. We assume that the low energy effects of LV can be parameterized in terms of a local EFT. Furthermore, for simplicity we assume that only boost invariance is broken, while rotations are preserved (see [22] for further comments on rotation breaking in this context). Therefore we introduce LV by coupling standard model fields to a non-zero vector. More complicated forms could of course be chosen, however this would introduce significant direction dependence. With low statistics already for neutrino observatories, disentangling direction dependence would be a difficult task.

We focus on the CPT even mass dimension five and six operators involving a vector field $u^{a}$ (which we assume to describe the preferred reference frame in which the CMB is seen as isotropic) coupled to a Dirac neutrino $\psi$ in a mass eigenstate with mass $m$ that are quadratic in matter fields and hence modify the free field equations. Neglecting the left-handed helicity of neutrinos for a moment, the Lagrangian for a generic fermion is the usual Dirac term plus [19]

$$
\begin{aligned}
& \bar{\psi}\left[-\frac{1}{M_{\mathrm{Pl}}}(u \cdot D)^{2}\left(\alpha_{L}^{(5)} P_{L}+\alpha_{R}^{(5)} P_{R}\right)\right. \\
& -\frac{i}{M_{\mathrm{Pl}}^{2}}(u \cdot D)^{3}(u \cdot \gamma)\left(\alpha_{L}^{(6)} P_{L}+\alpha_{R}^{(6)} P_{R}\right)
\end{aligned}
$$




$$
\left.-\frac{i}{M_{\mathrm{Pl}}^{2}}(u \cdot D) \square(u \cdot \gamma)\left(\tilde{\alpha}_{L}^{(6)} P_{L}+\tilde{\alpha}_{R}^{(6)} P_{R}\right)\right] \psi,
$$

where $u^{a}$ is a timelike unit vector describing the preferred frame, $P_{R}$ and $P_{L}$ are the usual right and left projection operators, $P_{R, L}=\left(1 \pm \gamma^{5}\right) / 2$, and $D$ is the gauge covariant derivative. The $\alpha$ coefficients are dimensionless.

For fermions, at $E \gg m$ the helicity eigenstates are almost chiral, with mixing due to the particle mass and the dimension five operators. Since we will be interested in high energy states, we re-label the $\alpha$ coefficients by helicity, i.e. $\alpha_{+}^{(d)}=\alpha_{R}^{(d)}, \alpha_{-}^{(d)}=\alpha_{L}^{(d)}$. The resulting high energy dispersion relation for positive and negative helicity particles can easily be seen from (1) to involve only the appropriate $\alpha_{+}^{(d)}$ or $\alpha_{-}^{(d)}$ terms. For compactness, we denote the helicity based dispersion by $\alpha_{ \pm}^{(d)}$. Therefore at high energies we have the dispersion relation

$$
E^{2}=p^{2}+m^{2}+f_{ \pm}^{(4)} p^{2}+f_{ \pm}^{(6)} \frac{p^{4}}{M_{\mathrm{Pl}}^{2}}
$$

where $f_{ \pm}^{(4)}=\frac{m}{M_{\mathrm{Pl}}}\left(\alpha_{-}^{(5)}+\alpha_{+}^{(5)}\right)$ and $f_{ \pm}^{(6)}=2 \alpha_{ \pm}^{(6)}+\alpha_{-}^{(5)} \alpha_{+}^{(5)}$. We have dropped the $\tilde{\alpha}_{R, L}^{(6)}$ terms as the $\square$ operator present in these terms makes the correction to the equations of motion proportional to $m^{2}$ and hence tiny.

The dimension five fermion operators induce two corrections, one proportional to $E^{4}$ and one corresponding to a change in the limiting speed of the fermion away from c. The second effect, generated by $f^{(4)}$ is naturally of order $10^{-30}$ due to the mass suppression for neutrinos and hence can also be disregarded. Furthermore, we can drop the positive helicity coefficients, as the dominant signal will be from left-handed neutrinos produced by standard model couplings. Finally, since CPT is conserved, and neutrinos and antineutrinos exist only in opposite states of helicity, $f_{\nu}^{(6)} \equiv \eta_{\nu}=f_{\bar{\nu}}^{(6)}$. All these lovely properties of neutrinos mean we have just one LV coefficient, while in other cases [26] further assumptions, such as parity preservation, are needed to reduce the number of free parameters. The final dispersion relation we assume in this work for each mass eigenstate neutrinos and antineutrinos is

$$
E_{\nu}^{2}=p^{2}+m_{\nu}^{2}+\eta_{\nu I} \frac{p^{4}}{M_{\mathrm{Pl}}^{2}}
$$

where $I$ denotes the mass eigenstate of the neutrino. There is no reason why each mass eigenstate needs have the same coefficient, and indeed whether or not they do makes a dramatic difference in the observed spectrum. In particular, we will study two cases: in the "flavor blind" case all the $\eta_{\nu I}$ have roughly the same magnitude, which translates in all the LV effects being essentially independent of flavor; in the "flavor dependent" case the $\eta_{\nu I}$ are instead different for different mass states, which makes the LV effects depend upon flavor states. Among the "flavor blind" cases we can find a "best case" scenario, in which the effects of LV are maximal, as well as a "worst case" scenario, in which LV is present but is ineffective in the context of UHE neutrinos we are discussing now.

A fairly accurate general estimate of the minimum energy in which LV corrections in equation (3) is relevant is obtained, as stated previously, by comparing the largest mass 
of the particles entering in the LV reaction with the magnitude of the LV correction in these equations [37]. In our case, assuming $\eta_{\nu I} \sim 1$, the typical energy at which $\mathrm{LV}$ contributions start to be relevant is of order $E_{t h} \sim \sqrt{m_{\nu} M_{\mathrm{Pl}}} \simeq 10^{13} \mathrm{eV}$, assuming $m_{\nu} \simeq 10^{-2} \mathrm{eV}$. This energy of $10 \mathrm{TeV}$ allows observatories such as ICECUBE to possibly constrain these operators in the future using neutrino oscillations. We instead focus on changes to the UHE neutrino spectrum induced by these operators.

\section{UHE neutrinos and LV}

If we neglect exotic sources of UHE neutrinos (as suggested in many top-down models for the production of UHECRs, now disfavored by the current experimental photon limits), the "cosmogenic" neutrino flux is created [53, 54, 55, 56] via the decay of charged pions produced by the interaction of primary nucleons with CMB photons above $E_{p r} \simeq 5 \times 10^{19} \mathrm{eV}$, the Greisen-Zatsepin-Kuz'min (GZK) effect [57]. HiRes [58] and AUGER [59] spectral observations seem to confirm the presence of a GZK suppression in the UHECR spectrum. Although the suppression of the UHECR spectrum could be also due to the maximal accelerating power of UHECR sources, the fact that it occurs at just the right energy for being GZK taking place during propagation, and the results 60. on the correlation of the UHECR arrival directions with the large scale distribution of matter within $\sim 75 \mathrm{Mpc}$, seem to favor the GZK explanation. Motivated by these considerations, we make here the hypothesis that the GZK reaction is at work during UHECR propagation.

We have checked numerically that the process of pion decay is not strongly affected by the above LV if only neutrinos are LV. One might ask in general if LV effects in the hadronic sector, which we have so far neglected, can matter in the production of UHE neutrinos. We can neglect the hadronic sector because of the tight constraints already placed on such LV operators [27, 28, 29, 26], which are stronger than what will be considered here.

Violation of Lorentz invariance however introduces new phenomena in the propagation of UHE neutrinos. A partial list of these effects includes:

Modified $\nu$ oscillations Since the LV is parameterized in the mass eigenstate, the LV terms act as contributions to an effective mass and contribute to neutrino oscillations. Cosmogenic neutrinos are not the right phenomena with which to study modified oscillations as they have oscillated many times during their flight from an unknown source, making it extremely hard to derive oscillation constraints. ICECUBE and other neutrino observatories may be sensitive to these operators for atmospheric neutrinos.

$\nu$-Čerenkov Čerenkov radiation in vacuum via charge-radius coupling or gravity: $\nu \rightarrow \nu \gamma$. This possibility has been already investigated for renormalizable operators [46, 40]. The rate is too small for both renormalizable operators (at current limits) or non-renormalizable operators, even for cosmogenic neutrinos. It also involves additional LV coefficients for photon emission and perhaps new modes 
for graviton emission [61, thereby complicating the analysis and, at best, yielding less definitive limits.

$\nu$-splitting : $\nu \rightarrow \nu \nu \bar{\nu}$. This effect is what we focus on in the following, as it exclusively involves the neutrino sector and has a high enough rate to be seen at UH energies.

$\nu$-pair emission : $\nu \rightarrow \nu Z \rightarrow \nu f \bar{f}$. Here, $f$ represents some other fermion species besides neutrinos. Electrons are the only fermion species light enough to have an effect compared to $\nu$-splitting at $\mathrm{UH}$ energies. We initially will ignore this reaction, but we will consider the possible effects of electron pair and hadronic emission in section 6 .

\section{Neutrino Splitting}

To understand how a neutrino can split into three, it suffices to calculate the threshold energy for this reaction to occur, which in a Lorentz invariant scenario would be infinite. Let us compute the threshold for the decay $\nu_{A}(p) \rightarrow \nu_{A}\left(p^{\prime}\right) \nu_{B}(q) \bar{\nu}_{B}\left(q^{\prime}\right)$, where $\nu_{A}, \nu_{B}$ are neutrinos of mass $m_{A}, m_{B}$ and $p, p^{\prime}, q, q^{\prime}$ are the momenta. In the threshold configuration the momenta of the outgoing particles are all aligned and parallel to the direction of the incoming neutrino momentum [62], hence we choose all momenta to be in the $z$-direction. Let $x, y$ and $t$ be the fraction of initial momentum carried respectively by the outgoing $\nu_{A}$, by $\nu_{B}$, and by $\bar{\nu}_{B}, t=1-x-y$ and $0<x, y, t<1$. For a general dispersion relation with LV term $\eta^{(n)} p^{n} / M_{\mathrm{Pl}}^{n-2}$ the threshold equation (the energy conservation equation imposing momentum conservation) can then be written as

$$
\frac{p^{n}}{M_{\mathrm{Pl}}^{n-2}}\left[\eta_{\nu_{A}}^{(n)}\left(1-x^{n-1}\right)-\eta_{\nu_{B}}\left(y^{n-1}+t^{n-1}\right)\right]=m_{\nu_{A}}^{2} \frac{1-x}{x}+m_{\nu_{B}}^{2}\left(\frac{1}{y}+\frac{1}{t}\right)
$$

When LI is exact, the left hand side vanishes and there is no solution. With LV, in the simple case where $n=4$ and $A=B$, we obtain the equation

$$
\frac{p^{4}}{M_{\mathrm{Pl}}^{2} m_{\nu_{A}}^{2}} \eta_{\nu_{A}}^{(4)}=\frac{1}{3 x y t}
$$

The minimum of the right-hand side in the equation above is 9 and is attained at $x=y=t=1 / 3$, hence the threshold energy is given by

$$
p_{\mathrm{th}(4)}^{\nu \rightarrow \nu \bar{\nu}}=\sqrt{\frac{3 M_{\mathrm{Pl}} m_{\nu_{A}}}{\left(\eta_{\nu_{A}}^{(4)}\right)^{1 / 2}}} \simeq 20 \mathrm{TeV} \sqrt{\frac{m_{\nu_{A}}}{10^{-2} \mathrm{eV}\left(\eta_{\nu_{A}}^{(4)}\right)^{1 / 2}}} .
$$

Note that $\eta_{\nu_{A}}$ must be positive in order for there to be splitting, i.e. we must deal with superluminal neutrinos. If $A \neq B$ then the threshold energy must be solved for numerically as the LV parameter and mass are generically different. However, the existence of a finite threshold can still be shown for appropriate values of $\eta_{\nu_{A}}$ and $\eta_{\nu_{B}}$. In the latter case, the coefficients do not need to be all positive for there to be a threshold, however it is necessary that $\eta_{\nu_{B}}<\eta_{\nu_{A}}-K(p)$, where $K(p)$ is a positive number that depends on the incoming momentum. Thresholds for negative coefficients have been 
investigated previously [37, 63] and there are strong limits on the LV coefficients 4 However, for UHE neutrinos it is more difficult to extract constraints, as we shall discuss in Section 5.2 .

\subsection{The best case scenario: flavor blind $L V$}

Neutrinos created in a flavor eigenstate are, of course, a combination of mass eigenstates. In order to maximize the neutrino splitting signal one would want all mass eigenstates to split ("flavor blind" scenario). The best case scenario is therefore when $\eta_{\nu I}>0$ for each $I$ and each $\eta_{\nu I}$ is of the same order of magnitude (so all states begin to decay at the same energy). As our best case scenario then we take $\eta_{\nu I}$ equal and positive for all $I$.

4.1.1. Decay time computation Let us now consider, within the above "flavor blind scenario", energies well above threshold so that neutrinos do effectively split, and the best case combination of the $\eta_{\nu I}$. The splitting rate can be calculated from the neutrino width

$$
\Gamma_{t o t, A}=\sum_{\mathrm{B}} \Gamma_{A B}
$$

where the sum runs over the open splitting channels and $\Gamma_{A B}$ represents the partial width for the channel $\nu_{A} \rightarrow \nu_{A} \nu_{B} \bar{\nu}_{B}$. $\Gamma_{A B}$ is simply

$$
\Gamma_{A B}=\frac{1}{2 E_{p}} \times \int\left|\mathcal{M}_{A B}\right|^{2} \times d \Phi_{A B}
$$

where $\Phi_{A B}$ represents the phase space of the final states and $\mathcal{M}_{A B}$ the matrix element for the process. The dominant channel for neutrino splitting is via the tree level neutral current interaction. The matrix element for this interaction is

$$
\mathcal{M}_{A B} \propto \frac{g^{2}}{4 \cos ^{2} \theta_{w}} \bar{u}_{A}\left(p^{\prime}\right) \gamma^{\mu} P_{L} u_{A}(p) \frac{g_{\mu \nu}}{r^{2}-M_{Z}^{2}+i M_{Z} \Gamma_{Z}} \bar{u}_{B}\left(q^{\prime}\right) \gamma^{\nu} P_{L} v_{B}(q),
$$

where $g$ is the charged current electro-weak coupling constant, $P_{L}$ is the usual spin projector, $\theta_{w}$ is the Weinberg angle and $r^{\mu}$ represent the 4-momentum components of the $Z$ boson. $u$ and $v$ are the spinors associated with $\nu_{A}$ and $\nu_{B}$, their functional form can be found in [19]. $r^{2}$ is at most the order of $p^{4} / M_{P l}^{2}$ and hence for any incoming momenta $p<10^{19.5} \mathrm{eV} r^{2} \ll M_{Z}^{2}$. After some brute force algebra, we end with

$$
\mathcal{M}_{A B} \propto \frac{g^{2}}{4 M_{Z}^{2} \cos ^{2} \theta_{w}} \sqrt{16 E_{p} E_{p^{\prime}} E_{q} E_{q^{\prime}}} \times F(\chi),
$$

where $\chi$ is the angle between incoming and outgoing $\nu_{A}$ and $F(\chi)$ is a complicated function of $\chi$.

I We notice here that according to our eq. (4) neutrino splitting is forbidden for superluminal neutrinos in the case $n=1$ studied e.g. in 64. Although the model presented in 64 does not have an EFT description, a simple threshold computation under the assumption of energy-momentum conservation and equal values of masses and LV parameters for neutrinos and antineutrinos shows that if neutrino splitting is possible also in that framework, values $\eta_{\nu}^{(1)} \sim-10^{-51}$ can be probed by $\sim 10^{19} \mathrm{eV}$ neutrinos. 
The phase space, $d \Phi_{A B}$ is given by

$$
d \Phi_{A B}=(2 \pi)^{4} \delta^{(4)}\left(p_{\text {in }}-\sum p_{\text {out }}\right) \frac{d^{3} p^{\prime}}{(2 \pi)^{3} 2 E_{p^{\prime}}} \frac{d^{3} q^{\prime}}{(2 \pi)^{3} 2 E_{q^{\prime}}} \frac{d^{3} q}{(2 \pi)^{3} 2 E_{q}} .
$$

We integrate over $q^{\prime}$ and we are left with

$$
d \Phi_{A B}=\frac{1}{8(2 \pi)^{5}} \delta\left(E_{p}-E_{p^{\prime}}-E_{q}-E_{q^{\prime}}\right) \frac{d^{3} p^{\prime} d^{3} q}{E_{p^{\prime}} E_{q} E_{q^{\prime}}} .
$$

Substituting $M_{A B}$ and $d \Phi_{A B}$ back into (8) we have

$$
\Gamma_{A B}=\frac{g^{4}}{16(2 \pi)^{5} M_{Z}^{4} \cos ^{4} \theta_{w}} \int \delta\left(E_{p}-E_{p}^{\prime}-E_{q}-E_{q}^{\prime}\right) d^{3} p^{\prime} d^{3} q F^{2} .
$$

We now turn to estimating the size of the remaining integral well above threshold, as UHE neutrinos are far in excess of the neutrino splitting threshold around $20 \mathrm{TeV}$. Temporarily, we will set $\eta_{\nu}=1$ and re-insert it at the end. We first assume that the opening angle for all three neutrinos is roughly equal, as is true over most of phase space. In this case, $F(\chi)$ can be approximated as $(1-\cos \chi)$. The opening angle $\chi$ vanishes as $M_{P l} \rightarrow \infty$, and is small even for UHE neutrinos. $F(\chi)$ then reduces to $F(\chi)=\chi^{2} / 2$. Indeed, $\chi$ is given by the typical transverse momenta $p_{\perp}$ divided by the longitudinal momenta $p_{\|}$. The characteristic size of $p_{\perp}$ can be estimated by energy conservation, recognizing that when energy is conserved any "excess" energy from LV goes into the energy needed to create transverse momenta. Formally this implies that

$$
\frac{p_{\|}^{3}}{M_{\mathrm{Pl}}^{2}} \sim \frac{p_{\perp}^{2}}{p_{\|}},
$$

i.e. $p_{\perp} \sim p_{\|}^{2} / M_{\mathrm{Pl}}$. $p_{\|}$itself for any particle can range from almost 0 to almost the initial energy $E_{p}$ well above threshold. The available phase space volume for the remaining outgoing particles can therefore be approximated (although somewhat overestimated) as a cylinder with length $E_{p}$ and radius $E_{p}^{2} / M_{\mathrm{Pl}}$, which has a total volume of $\pi E_{p}^{5} / M_{\mathrm{Pl}}^{2}$. Similarly, $\chi$ can be estimated as $\chi \sim p_{\|} / M_{\mathrm{Pl}} \sim E_{p} / M_{\mathrm{Pl}}$. The $\delta$-function in energy simply removes one factor of energy from our equation. Putting these expressions back into (13) we find

$$
\Gamma_{A B} \sim \frac{g^{4}}{16(2 \pi)^{5} M_{Z}^{4} \cos ^{4} \theta_{w}} \frac{1}{E_{p}}\left(\frac{\pi E_{p}^{5}}{M_{\mathrm{Pl}}^{2}}\right)^{2} \frac{E_{p}^{4}}{4 M_{\mathrm{Pl}}^{4}},
$$

or, simplified

$$
\Gamma_{A B} \sim \frac{G_{F}^{2}}{64 \pi^{3} M_{\mathrm{Pl}}^{8}} E^{13}
$$

where we used $G_{F} / \sqrt{2}=g^{2} /\left(8 M_{Z}^{2} \cos ^{2} \theta_{w}\right)$. If we now want to restore the $\eta_{\nu}$ factor, we notice that we must put one $\eta_{\nu}$ for each $M_{\mathrm{Pl}}^{-2}$. In the end, the total rate is computed by adding the partial rates for each neutrino flavor, which is equivalent to multiply by 3. Hence, we have

$$
\Gamma_{\nu \nu \bar{\nu}} \sim \frac{3 G_{F}^{2}}{64 \pi^{3}} \frac{\eta_{\nu}^{4} E^{13}}{M_{\mathrm{Pl}}^{8}}
$$


This width can be turned into a decay length as

$$
L_{\nu \nu \bar{\nu}}=\frac{c}{\Gamma_{\nu \nu \bar{\nu}}} \sim 1.7 \times 10^{-3} \operatorname{Mpc} \eta_{\nu}^{-4}\left(\frac{E}{10^{19} \mathrm{eV}}\right)^{-13} .
$$

This makes clear we need to push the required energies above $10^{18.5} \mathrm{eV}$ (with $\eta_{\nu}=1$ ) for the rate to be appreciable. As a final remark, we notice that the decay length in Eq. (18) strongly depends on both the energy and $\eta_{\nu}$. Therefore, the error about its actual magnitude we might have made in our estimate will reflect in very small errors in the determination of the energy at which LV effects start to be relevant as well as of the constraint on $\eta_{\nu}$.

4.1.2. $Z$ boson resonance At such high energies the $\mathrm{Z}$ could be real - i.e. there is a resonance in the matrix element. Even in this regime, however, the neutrino decay time can be computed easily, as the only hypothesis one has to relax is that the $\mathrm{Z} 4$ momentum $r$ satisfies $r^{2} \ll M_{Z}^{2}$. The magnitude of $r^{2}$ can be easily computed exploiting the kinematic equations. We obtain $r^{2}=16 / 27 \eta_{\nu} E_{\nu}^{4} / M_{\mathrm{Pl}}^{2}$. The final decay length is then

$L_{\nu \nu \bar{\nu}} \sim 1.7 \times 10^{-3} \mathrm{Mpc} \eta_{\nu}^{-4}\left(\frac{E_{\nu}}{10^{19} \mathrm{eV}}\right)^{-13} \times\left[\left(1-\frac{16}{27} \eta_{\nu} \frac{E^{4}}{M_{\mathrm{Pl}}^{2} M_{Z}^{2}}\right)^{2}+\left(\frac{\Gamma_{Z}}{M_{Z}}\right)^{2}\right]$.

A comparison between the two different decay lengths, Eq. (18) and Eq. (19), can be found in Fig. 1. The $\mathrm{Z}$ resonance is hit at $E^{4}=27 / 16\left(\eta_{\nu}^{-1} M_{\mathrm{Pl}}^{2} M_{Z}^{2}\right)$. We notice that even

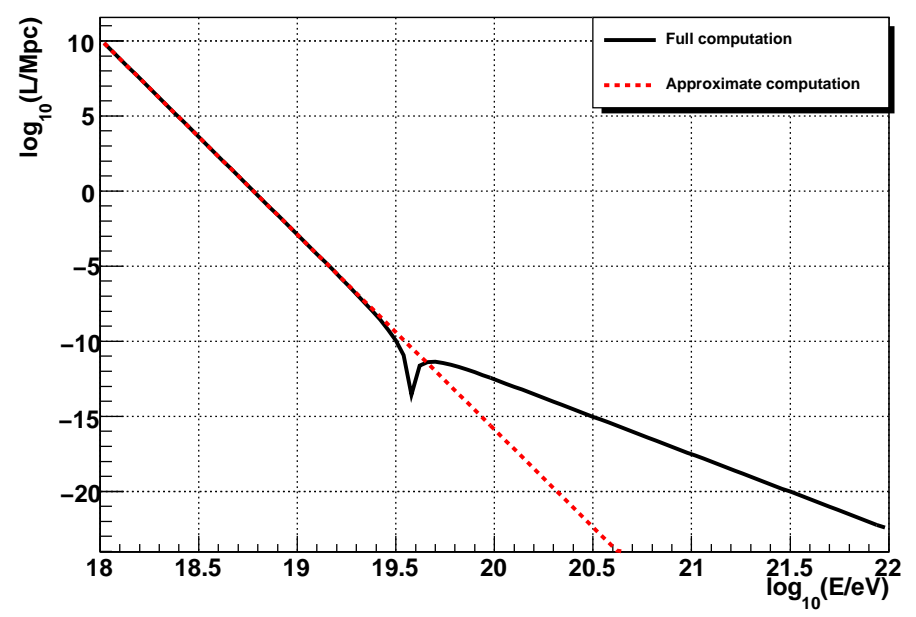

Figure 1. Comparison between computations of the decay length without (Eq. (18)/red dashed line), and with (Eq. (19)/black solid line) the Z boson resonance.

though the two computations lead to very different results above the resonance, they will not lead to any appreciable effect in the neutrino spectra, as at such energies the decay lengths are anyway much smaller than the propagation distance of cosmological neutrinos. 


\subsection{A non-optimal case: flavor dependent $L V$}

In the flavor blind case above every mass eigenstate undergoes splitting. It is easy to construct a scenario where only one mass eigenstate decays - one eigenstate has a positive $\eta \mathrm{LV}$ coefficient while the other two have no LV. While this is unnatural in some sense, it serves as a nice example to illuminate a method to easily hide LV.

The neutrino spectrum produced by the GZK process has a distinct distribution in flavor states of $1 / 3 \nu_{e}$ and $2 / 3 \nu_{\mu}$. Therefore, in order to calculate a spectrum with LV we need to convert this flavor spectrum into a mass spectrum, i.e. we need to choose a neutrino mixing model. For our purposes we choose tribimaximal mixing [73, 39], which satisfies current experimental constraints and provides a simple mixing matrix. With tribimaximal mixing the GZK neutrino spectrum has equal distribution over all mass eigenstates.

Now that we have spelled out out test models we shall proceed discussing the results for both scenarios.

\section{Results}

The net effect of neutrino splitting is very simple. It kills one neutrino of energy $E$ and creates 3 neutrinos of average energy $E / 3$, provided the parent neutrino is above threshold and has a reasonable life time. As we showed in sec. 4.1.1, for a life time shorter than the age of the Universe, the neutrino energy has to be above $10^{18.5} \mathrm{eV}$, i.e. we need to probe UHE neutrinos.

The effects of neutrino splitting on the UHE neutrino spectrum are twofold and can be understood qualitatively as follows.

Flux suppression at $\mathbf{U H}$ energies The splitting is effectively an energy loss process for UHE neutrinos. If the rate is sufficiently high, the energy loss length can be below $1 \mathrm{Mpc}$. Let us call $\bar{E}\left(\eta_{\nu}\right)$ the energy at which this happens. Then, being GZK neutrinos produced mainly at distances larger than $1 \mathrm{Mpc}$, we do not expect any neutrino to be detected at Earth with $E>\bar{E}$.

The mere observation of neutrinos up to a certain energy $E_{\text {obs }}$ would imply a constraint, according to Eq. 18

$$
\eta_{\nu}^{(4)} \lesssim\left(\frac{E_{\mathrm{obs}}}{6 \times 10^{18} \mathrm{eV}}\right)^{-13 / 4}
$$

Flux enhancement at sub-UH energies Neutrinos lose energy by producing lower energy neutrinos. Eventually these neutrinos will become stable, either because their energy is below threshold, or because their lifetime is larger than their propagation time. Accordingly, we expect an enhancement of the neutrino flux at energies below few $\times 10^{18} \mathrm{eV}$.

In spite of being qualitatively straightforward, however, this analysis is not powerful enough to provide us with constraints on LV. In order to obtain meaningful constraints, 
we have to resort to full MonteCarlo simulations of the UHECR propagation from sources to the Earth.

We simulated then the propagation of UHECR protons in the Inter Galactic Medium using the Monte Carlo package CRPropa [65], suitably modified to take into account LV in the neutrino sector. The simulation parameters are the following: we simulated unidimensional UHECR proton propagation, with source energy spectrum $d N / d E \propto E^{-2.2}$, from a spatially uniform distribution of sources located at redshift $z<3$ according to the Waxman \& Bahcall (WB) distribution used in [66]. The injection proton spectrum was tuned to fit AUGER data [59].

\subsection{Results for flavor bind LV scenario}

Figure 2 shows the outcome of the simulations for different values of the LV parameter $\eta_{\nu}$ in the best case scenario, together with experimental sensitivities from some existing and planned observatories, as well as the Waxman \& Bahcall bound [68, 69] for reference. Results are in agreement with qualitative expectations previously discussed: Above a

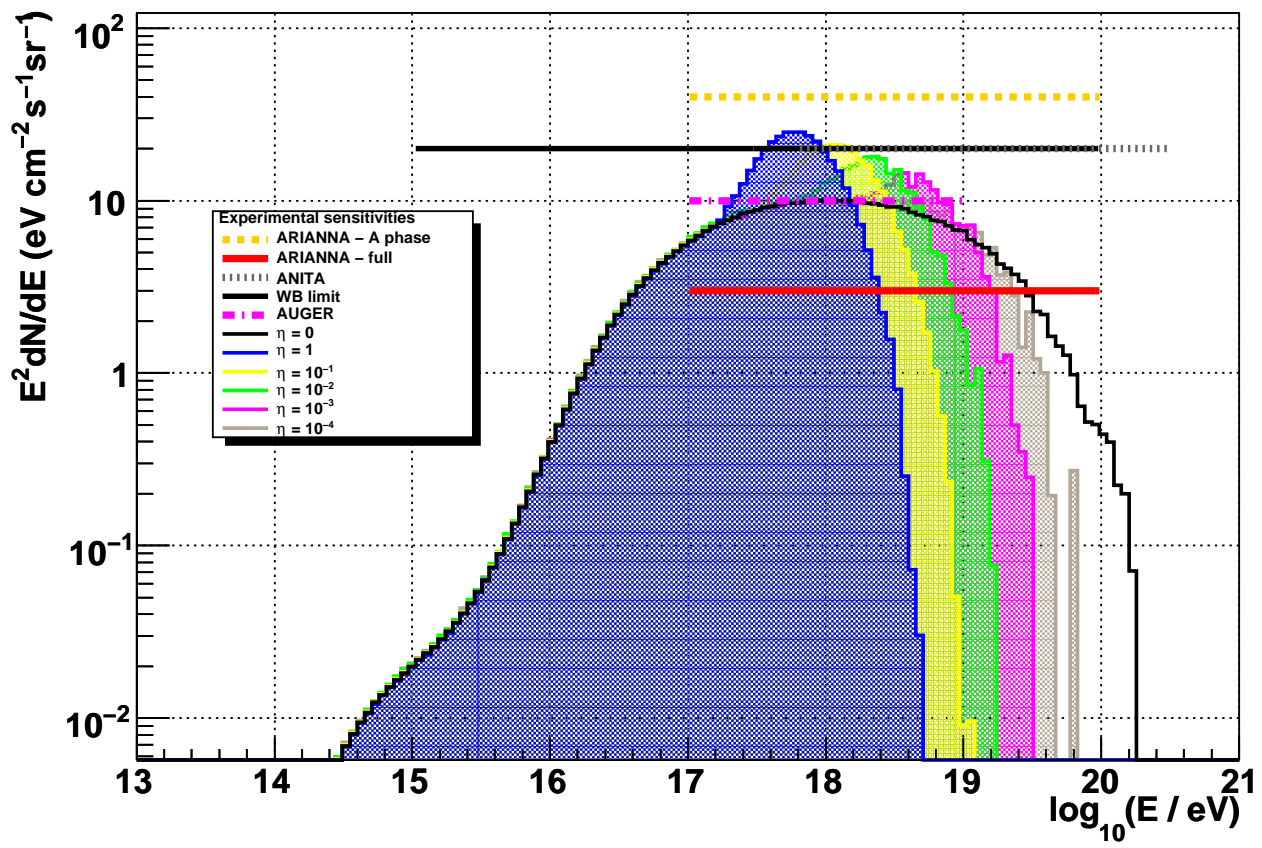

Figure 2. Evolution of the predicted LV neutrino spectra varying $\eta_{\nu}$ in the "best case scenario". Sensitivities of main UHE neutrino operating and planned experiments are shown, as found in [51, 47, 67]. The Waxman \& Bahcall limit [68, 69] in the interesting energy range is shown for reference.

+ This limit is in fact an estimate of the neutrino luminosity of sources of UHE Cosmic Rays and $\gamma$-rays, in the hypothesis that the sources are optically thin to the escape of UHE particles and that both $\gamma$-rays and neutrinos are originated from UHECR interactions with radiation backgrounds. It is worth mentioning that this bound might be strongly affected by QG effects, as shown in [70]. 
certain energy the neutrino spectrum displays a sharp cut off, while at lower energy a peak appears. The peak position and strength, as well as the position of the cut off, depend on the value of the LV parameter $\eta_{\nu}$. If $\eta_{\nu}=1$ the peak is found at $\lesssim 10^{18} \mathrm{eV}$ and overwhelms the LI spectrum by a factor of roughly 10, while the cut off energy is at $\sim 10^{18.7} \mathrm{eV}$. Also the evolution of the neutrino spectra with $\eta_{\nu}$ can be seen in the same Fig. 2.

Although at present it is not possible to draw firm conclusions for the constraints on LV in the neutrino sector, we notice that future experiments, if not already AUGER, will be able to probe the fluxes we predict, hence they will be able to cast constraints on $\eta_{\nu}$. In the future, the ARIANNA experiment [50] will be able to probe fluxes down to $\sim 3 \mathrm{eV} \mathrm{cm}^{-2} \mathrm{~s}^{-1} \mathrm{sr}^{-1}$ in the energy range $10^{17} \div 10^{20} \mathrm{eV}$ in the full configuration [51. If such a sensitivity will be achieved, a constraint of order $\eta_{\nu} \lesssim 10^{-4}$ will be cast, according to Fig. 2. Moreover, the IceRay experiment [52] planned at the South pole is expected to observe roughly 4 neutrino events per year of data taking for the WB model preferentially studied here, in the range $10^{17} \div 10^{19.5} \mathrm{eV}$ [52]. Hence, constraints of order $\eta \lesssim 10^{-3}$ are expected after few years of data taking by this experiment. We finally notice here that by exploiting this strategy nothing can be said about the case $\eta_{\nu}<0$

\subsection{Results for flavor dependent LV scenario}

If only one mass eigenstate decays, the energy momentum conservation equations, rates, and lifetimes all remain the same. We therefore can apply our MonteCarlo spectrum for this scenario to just any one of the mass eigenstates (given our assumption of tribimaximal mixing no neutrino mass eigenstate is preferred). The only difference is that only $1 / 3$ of the total flux undergoes neutrino splitting. The resulting spectrum is shown in Fig. 3. Note that even for $\eta=1$ the departure from the LI spectrum is significantly reduced, as one would expect, with the magnitude of the deviation at the peak being lowered to a $\sim 50 \%$ excess over the LI flux. Hence, with present sensitivity, absence of a signal in the neutrino spectrum does not mean that LV is ruled out for neutrinos, even for $O(1)$ positive coefficients. We notice that the cutoff feature present in the flavor blind case has now effectively disappeared. Then, observations of a UHE neutrino flux up to some maximal energy do not allow to draw conclusions about LV in this case scenario.

We now return to the question of negative coefficients and neutrino splitting. So far, in both our flavor blind and flavor dependent scenarios all the coefficients have been positive. As argued in section 4, neutrinos can split even with negative coefficients when the relation $\eta_{\nu_{B}}<\eta_{\nu_{A}}-K(p)$ holds. However, one can immediately see that therefore at least one of the mass eigenstates is always stable if the coefficients are negative. This implies that no equivalent of the "best case scenario" (all the mass eigenstates decay) is possible for negative $\eta_{\nu I}$ and that, consequently, the deviation from the LI spectrum will be suppressed by at least $1 / 3$. Therefore cosmogenic neutrinos are not effective probes 


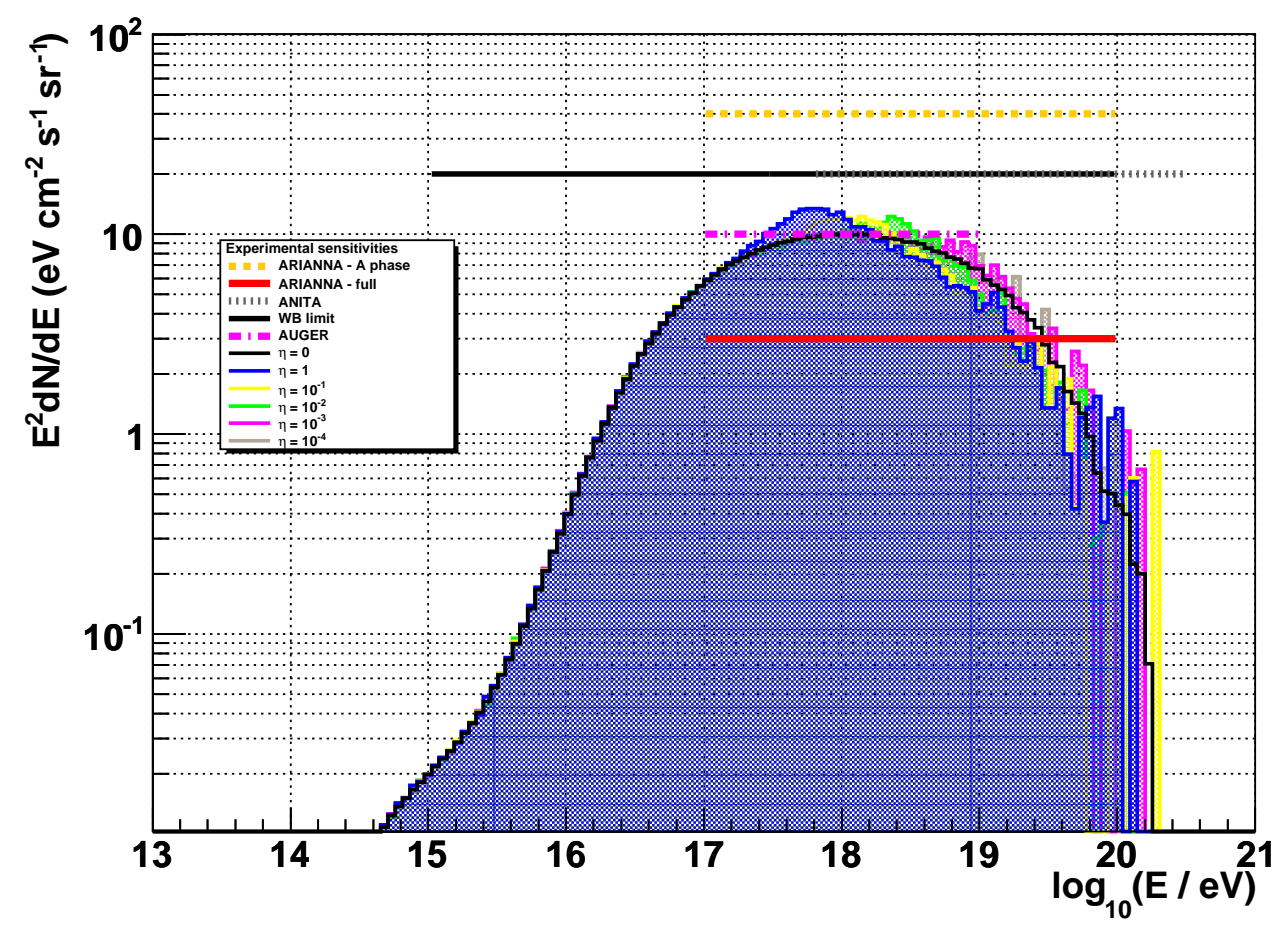

Figure 3. Observed UHE neutrino spectra for different values of the LV coefficient of the only mass eigenstate undergoing splitting in the non-optimal scenario. The flux suppression at UH energies has effectively disappeared (for what concerns observational relevance) and the excess at lower energies is significant only for $O(1) \mathrm{LV}$ coefficient.

of the $\eta_{\nu I}<0$ region of parameter space.

\subsection{Dependence on model uncertainties}

Predictions of cosmogenic neutrino spectra are known to be plagued by several uncertainties, as they depend strongly on the evolution of UHECR sources with redshift, which cannot be directly probed with UHECRs due to the GZK attenuation effect. As a consequence, also our predictions for the UHE neutrino flux in the presence of LV can be affected.

We check this by simulating different UHECR sources evolution models for the "best case scenario". In Fig. 4 we show the results for the model outlined in [56], which is expected to give the largest flux. As a result, the presence and the strength of the bump feature are demonstrated to be weakly dependent on the underlying UHECR source distribution, with the choice of the source distribution affecting by a factor of 2 the height of the bump. This was somewhat expected from our qualitative considerations: as long as UHECR sources are located at distances much larger than $1 \mathrm{Mpc}$ from Earth, the magnitude of the bump depends only on how many neutrinos were originally produced at energy larger than the LV cutoff energy. On the other hand, the cutoff feature is definitely model independent, as long as the unknown maximal energy at 


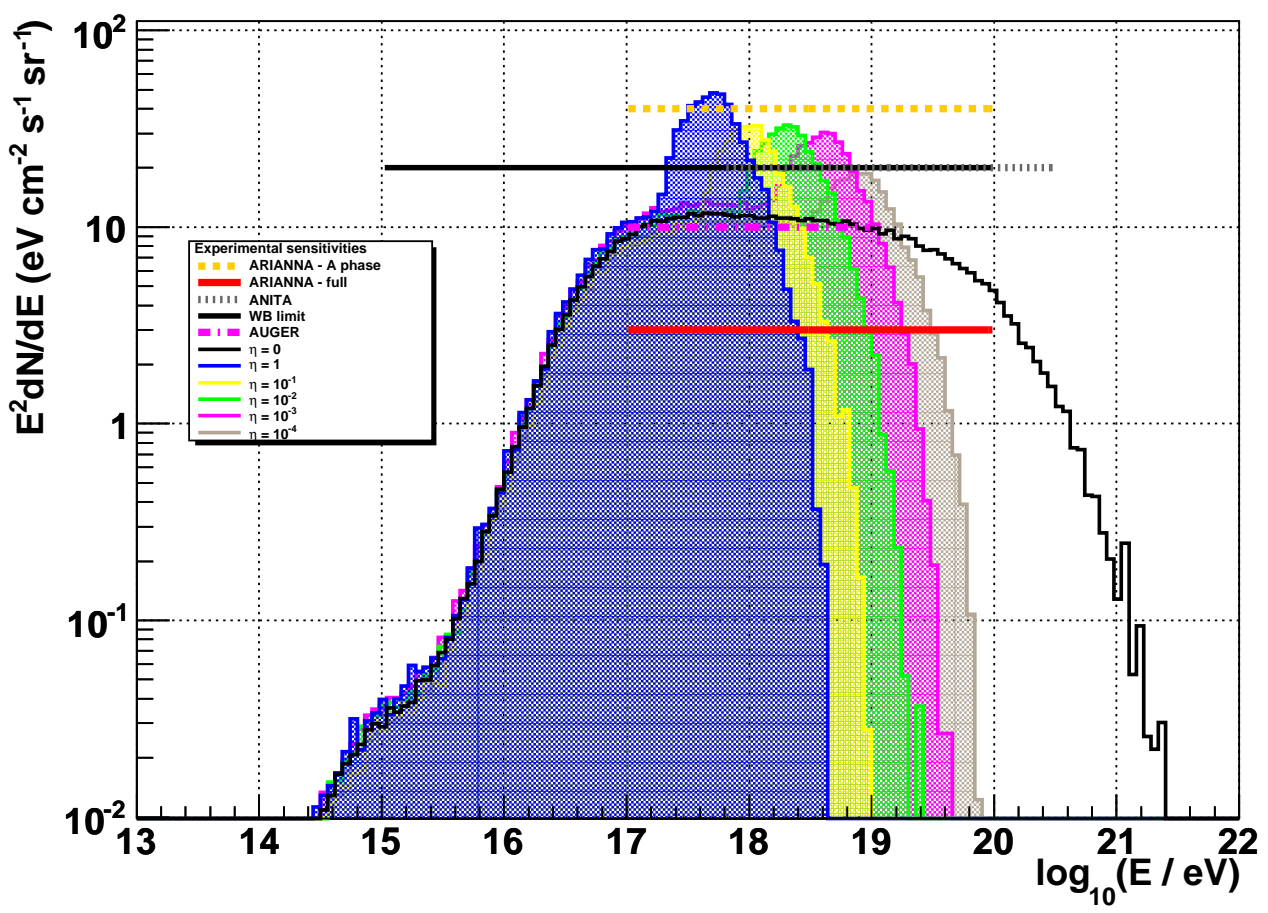

Figure 4. Evolution of the predicted LV neutrino spectra varying $\eta_{\nu}$ for the maximal model [56].

which UHECRs are accelerated is enough to produce neutrinos at energy larger than $\sim 6 \times 10^{18} \cdot \eta^{-4 / 13} \mathrm{eV}$.

\section{Other processes}

As we mentioned in the previous sections, there are other processes, besides splitting, leading to neutrinos losing energy in the inter-galactic medium. In particular, the hadronic decay modes of the $\mathrm{Z}^{0}$ could lead to the production of UHE protons, thereby mimicking a "Z-burst" effect [71]. Although this model was conceived for other purposes than constraining LV, and has now lost most of its attraction due to experimental results on proton spectra above the GZK threshold, it is notable that the same mechanism could in principle help constrain LV in the neutrino sector. However, we can argue with a very simple argument that this is not the case.

Let us consider the LI neutrino flux, which corresponds in Fig. 2 to the $\eta=0$ case. The minimal process leading to proton production in this context is $\nu \rightarrow \nu Z^{*} \rightarrow \nu p \bar{p}$, whose threshold energy, if $\eta_{\nu}=1$, is at $\sim 10^{18.7} \mathrm{eV}$. Processes involving more particles are naturally suppressed, however they represent the majority of the allowed channels and hence they might be relevant to our case. Even if we do not know the energy spectrum of the produced protons, we know that their mean energy is roughly $1 / 80$ times the energy of the parent neutrino [71]. We can then compute the maximal expected flux 
of UHECRs produced by this Z-burst-like mechanism by converting all the neutrinos with energy $E_{\nu}$ above threshold into 2 protons of energy $1 / 80 \times E_{\nu}$. The result is shown in Fig. 5. This mechanism would indeed contribute to the UHECR spectrum at

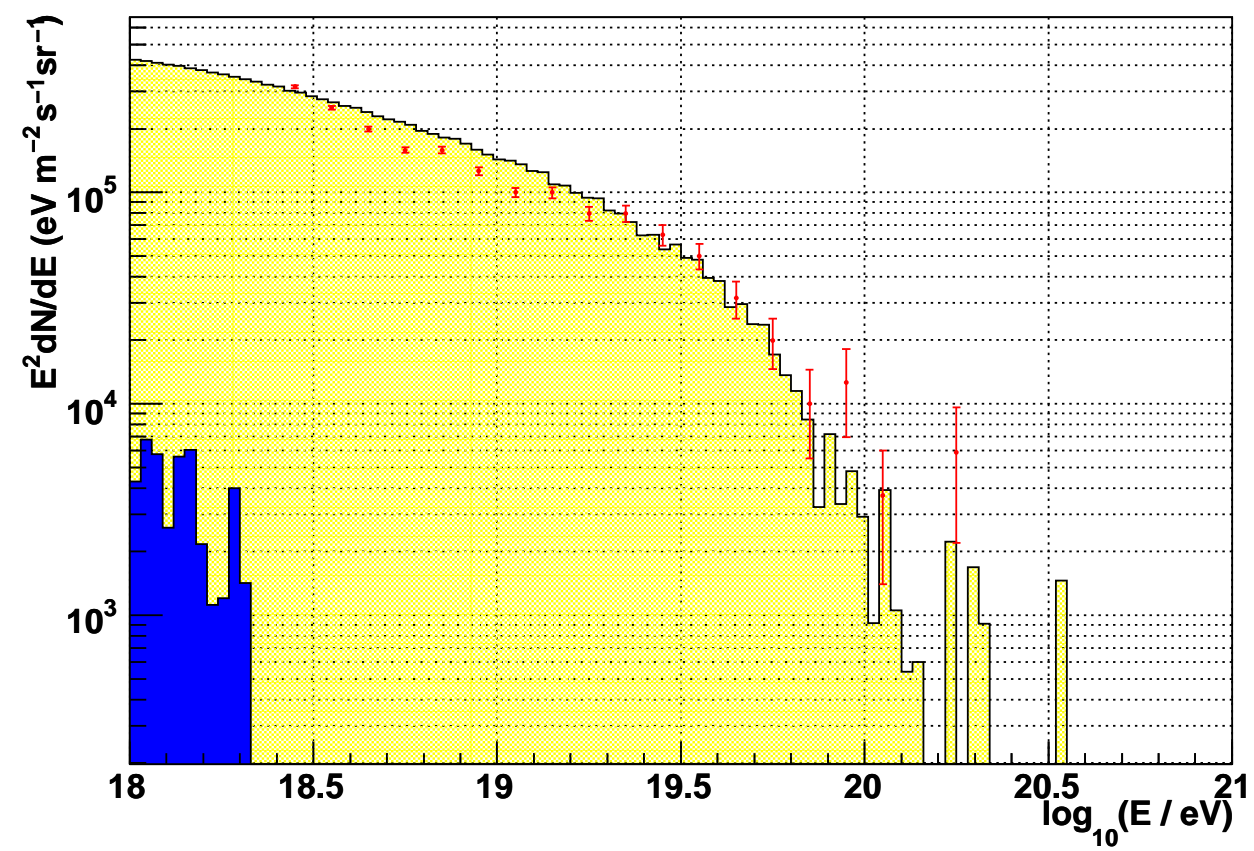

Figure 5. Observed UHE proton spectrum compared with predictions for just primary (yellow) and "Z-burst"-like originated protons (blue). The latter might produce an excess in the UHECR spectrum at $10^{18} \mathrm{eV}$, no larger than $5 \%$, and hence below the systematic uncertainties of UHECR experiments. Note that, in order to speed up computation, we switched off pair production, which is known to play an important rôle in reproducing the ankle feature [72. This is why our UHECR spectrum is above data between $10^{18.5} \mathrm{eV}$ and $10^{19} \mathrm{eV}$.

$10^{18} \mathrm{eV}$, producing an excess no larger than $5 \%$, which however is below the systematic uncertainties of UHECR experiments. Therefore, no constraints on LV can be implied using this technique.

Electron/positron pair production would also contribute to the cutoff in the neutrino spectrum. The threshold for pair production of electrons is at roughly $\sqrt{m_{e} M_{\mathrm{Pl}}}=10^{17} \mathrm{eV}$. At energies near the existing neutrino splitting cutoff, $10^{18.5} \mathrm{eV}$ we are well above threshold. The mass of the electrons is therefore irrelevant in calculating the decay rate (just as the neutrino mass was) and hence the electron pair production process has nearly the same rate as the neutrino splitting process. If neutrinos produce electron/positron pairs (which after all depends on LV in the electron sector) it can only increase the steepness of the cutoff. It will not contribute to the excess neutrino flux at $10^{17.5} \mathrm{eV}$ and hence one needs to probe energies one order of magnitude larger than $10^{17.5} \mathrm{eV}$ to derive constraints exploiting the presence or the absence of a cutoff feature. 


\section{Conclusions}

In this work we have investigated possible signals of higher dimension LV CPT even operators in the UHE neutrino spectrum, in particular the effect of the neutrino splitting on the UHE neutrino spectrum. This process provides a clean test as it does not involve other LV operators apart from the neutrinos' ones. In addition, since the dominant neutrino field is left-handed, there are no complications due to different LV for different chirality of fermion, as there are in the UHECR case, and one ends up with one LV coefficient for each neutrino mass eigenstate. In the flavor blind scenario, where every mass eigenstate undergoes splitting approximately at the same energy, there is both a precocious fall off of the neutrino flux at UHE as well as a significant excess in the UHE neutrino flux at energies as low as $10^{17.5} \mathrm{eV}$. Noticeably, this kind of energies are well within reach of current and future UHECR experiments and about order of magnitude below those so far used for LV tests with hadronic and electromagnetic UHECRs.

According to our study, existing or planned UHE neutrino experiments have the potential to probe $\mathrm{LV}$ in the neutrino sector for coefficients $\eta \gtrsim 10^{-4}$. However, we have discovered a serious difficulty with deriving constraints in the absence of a positive LV signal event. The distribution in mass eigenstates is roughly equal for UHE neutrinos in realistic mixing scenarios. Although it might seem somewhat unnatural that LV has different effects on different mass states of the same particle field, if this is the case then it is possible that LV can exist/be strong for one mass eigenstate yet be almost invisible for UHE neutrino detectors. In particular, the observation of a neutrino flux up to some maximal energy does not imply a firm conclusion on LV, at least with present accuracy. However, let us note that if a neutrino is ever detected at energy $E_{\nu}$, we can rule out a flavor blind $\eta_{\nu} \gtrsim\left(E_{\nu} / 10^{18.8} \mathrm{eV}\right)^{-13 / 4}$ according to Eq. (20). On the other hand, the bump feature can lead to constraints on $\eta_{\nu}>0$, as this bump should be observable in any such scenario of LV in the neutrino sector. In this case, to obtain a $O(1)$ constraint on $\mathrm{LV}$ requires at least a $50 \%$ accuracy in the determination of the neutrino spectrum in the energy range $10^{17} \div 10^{19} \mathrm{eV}$, which can be achieved by future experiments.

\section{Acknowledgments}

We thank J. Kelley and B. McElrath for useful discussions. This work was supported by the Deutsche Forschungsgemeinschaft through the collaborative research centre SFB 676 "Particles, Strings and the Early Universe: The Structure of Matter and SpaceTime". LM acknowledges support from the State of Hamburg, through the Collaborative Research program "Connecting Particles with the Cosmos" within the framework of the LandesExzellenzInitiative (LEXI).

\section{References}

[1] V. A. Kostelecky and S. Samuel, "Spontaneous Breaking Of Lorentz Symmetry In String Theory," Phys. Rev. D 39, 683 (1989). 
[2] J. R. Ellis, N. E. Mavromatos and D. V. Nanopoulos, Phys. Lett. B 665, 412 (2008) [arXiv:0804.3566 [hep-th]].

[3] R. Gambini and J. Pullin, "Nonstandard optics from quantum spacetime," Phys. Rev. D 59, 124021 (1999).

[4] C. Rovelli and S. Speziale, Phys. Rev. D 67, 064019 (2003) [arXiv:gr-qc/0205108].

[5] J. Alfaro and G. Palma, Phys. Rev. D 67 (2003) 083003 [arXiv:hep-th/0208193].

[6] S. M. Carroll, J. A. Harvey, V. A. Kostelecky, C. D. Lane and T. Okamoto, "Noncommutative field theory and Lorentz violation," Phys. Rev. Lett. 87, 141601 (2001). [arXiv:hep-th/0105082].

[7] J. Lukierski, H. Ruegg and W. J. Zakrzewski, Annals Phys. 243 (1995) 90 [arXiv:hep-th/9312153].

[8] G. Amelino-Camelia and S. Majid, Int. J. Mod. Phys. A 15 (2000) 4301 [arXiv:hep-th/9907110].

[9] M. Chaichian, P. P. Kulish, K. Nishijima and A. Tureanu, Phys. Lett. B 604, 98 (2004) [arXiv:hepth/0408069].

[10] G. Amelino-Camelia, J. R. Ellis, N. E. Mavromatos, D. V. Nanopoulos and S. Sarkar, "Potential Sensitivity of Gamma-Ray Burster Observations to Wave Dispersion in Vacuo," Nature 393, 763 (1998). [arXiv:astro-ph/9712103].

[11] C. P. Burgess, J. Cline, E. Filotas, J. Matias and G. D. Moore, "Loop-generated bounds on changes to the graviton dispersion relation," JHEP 0203, 043 (2002). [arXiv:hep-ph/0201082].

[12] C. Barcelo, S. Liberati and M. Visser, "Analogue gravity," Living Rev. Rel. 8, 12 (2005). [arXiv:grqc/0505065].

[13] P. Horava, Phys. Rev. D 79, 084008 (2009) [arXiv:0901.3775 [hep-th]].

[14] G. Amelino-Camelia, arXiv:0806.0339 [gr-qc].

[15] G. Amelino-Camelia, Int. J. Mod. Phys. D 11 (2002) 35 [arXiv:gr-qc/0012051].

[16] D. Colladay and V. A. Kostelecky, Phys. Rev. D 58, 116002 (1998) [arXiv:hep-ph/9809521].

[17] R. C. Myers and M. Pospelov, Phys. Rev. Lett. 90 (2003) 211601 [arXiv:hep-ph/0301124].

[18] P. A. Bolokhov and M. Pospelov, Phys. Rev. D 77, 025022 (2008) [arXiv:hep-ph/0703291].

[19] D. Mattingly, PoS QG-PH, 026 (2007).

[20] V. A. Kostelecky and M. Mewes, Phys. Rev. D 80 (2009) 015020 [arXiv:0905.0031 [hep-ph]].

[21] S. Groot Nibbelink and M. Pospelov, Phys. Rev. Lett. 94, 081601 (2005) [arXiv:hep-ph/0404271].

[22] D. Mattingly, Living Rev. Rel. 8, 5 (2005) [arXiv:gr-qc/0502097].

[23] T. Jacobson, S. Liberati and D. Mattingly, Annals Phys. 321 (2006) 150 [arXiv:astro-ph/0505267].

[24] S. Liberati and L. Maccione, arXiv:0906.0681 [astro-ph.HE]. To appear in Annual Review of Nuclear and Particle Science.

[25] J. Collins, A. Perez, D. Sudarsky, L. Urrutia and H. Vucetich, Phys. Rev. Lett. 93, 191301 (2004) [arXiv:gr-qc/0403053].

[26] L. Maccione, A. M. Taylor, D. M. Mattingly and S. Liberati, JCAP 0904 (2009) 022 [arXiv:0902.1756 [astro-ph.HE]].

[27] M. Galaverni and G. Sigl, Phys. Rev. Lett., 100, 021102 (2008). arXiv:0708.1737 [astro-ph].

[28] L. Maccione and S. Liberati, JCAP 0808, 027 (2008) [arXiv:0805.2548 [astro-ph]].

[29] M. Galaverni and G. Sigl, Phys. Rev. D 78 (2008) 063003 [arXiv:0807.1210 [astro-ph]].

[30] T. Kifune, Astrophys. J. 518 (1999) L21 [arXiv:astro-ph/9904164].

[31] R. Aloisio, P. Blasi, P. L. Ghia and A. F. Grillo, Phys. Rev. D 62 (2000) 053010 [arXiv:astro$\mathrm{ph} / 0001258]$.

[32] G. Amelino-Camelia and T. Piran, Phys. Rev. D 64 (2001) 036005 [arXiv:astro-ph/0008107].

[33] F. W. Stecker and S. T. Scully, Astropart. Phys. 23 (2005) 203 [arXiv:astro-ph/0412495].

[34] L. Gonzalez-Mestres, Nucl. Phys. Proc. Suppl. 190, 191 (2009) [arXiv:0902.0994 [astro-ph.HE]].

[35] S. T. Scully and F. W. Stecker, Astropart. Phys. 31 (2009) 220 [arXiv:0811.2230 [astro-ph]].

[36] F. W. Stecker and S. T. Scully, New J. Phys. 11 (2009) 085003 [arXiv:0906.1735 [astro-ph.HE]].

[37] T. Jacobson, S. Liberati and D. Mattingly, Phys. Rev. D 67 (2003) 124011 [arXiv:hep$\mathrm{ph} / 0209264]$.

[38] L. Maccione, S. Liberati, A. Celotti and J. G. Kirk, JCAP 0710 (2007) 013 [arXiv:0707.2673 [astro-ph]]. 
[39] Amsler W. M. et al. [Particle Data Group] Phys. Lett. B 667, 1 (2008).

[40] S. R. Coleman and S. L. Glashow, Phys. Rev. D 59 (1999) 116008 [arXiv:hep-ph/9812418].

[41] M. C. Gonzalez-Garcia, F. Halzen and M. Maltoni, Phys. Rev. D 71, 093010 (2005) [arXiv:hep$\mathrm{ph} / 0502223]$.

[42] J. S. Diaz, V. A. Kostelecky and M. Mewes, Phys. Rev. D 80, 076007 (2009) [arXiv:0908.1401 [hep-ph]].

[43] S. Yang and B. Q. Ma, arXiv:0910.0897 [hep-ph].

[44] M. C. Gonzalez-Garcia and F. Halzen, JCAP 0702 (2007) 008 [arXiv:hep-ph/0611359].

[45] A. Bhattacharya, S. Choubey, R. Gandhi and A. Watanabe, arXiv:0910.4396 [hep-ph].

[46] S. R. Coleman and S. L. Glashow, Phys. Lett. B 405 (1997) 249 [arXiv:hep-ph/9703240].

[47] P. Gorham et al. [ANITA collaboration], arXiv:0812.2715 [astro-ph].

[48] A. Petrolini, arXiv:0909.5220 [astro-ph.IM].

[49] A. Santangelo and A. Petrolini, arXiv:0909.5370 [astro-ph.HE].

[50] S. W. Barwick, J. Phys. Conf. Ser. 60 (2007) 276 [arXiv:astro-ph/0610631].

[51] S. W. Barwick, Nucl. Instrum. Meth. A 602 (2009) 279.

[52] P. Allison et al., arXiv:0904.1309 [astro-ph.HE].

[53] V. S. Beresinsky and G. T. Zatsepin, Phys. Lett. B 28 (1969) 423.

[54] F. W. Stecker, Astrophys. Space Sci. 20 (1973) 47.

[55] R. Engel, D. Seckel and T. Stanev, Phys. Rev. D 64 (2001) 093010 [arXiv:astro-ph/0101216].

[56] D. V. Semikoz and G. Sigl, JCAP 0404 (2004) 003 [arXiv:hep-ph/0309328].

[57] K. Greisen, Phys. Rev. Lett. 16 (1966) 748; Zatsepin, Kuz'min, Sov.Phys.JETP 4, 78.

[58] R. Abbasi et al. [HiRes Collaboration], arXiv:astro-ph/0703099.

[59] M. Roth [Pierre Auger Collaboration], arXiv:0706.2096 [astro-ph].

[60] J. Abraham et al. [Pierre Auger Collaboration], Science 318 (2007) 938 [arXiv:0711.2256 [astro$\mathrm{ph}]$.

[61] T. Jacobson and D. Mattingly, Phys. Rev. D 70 (2004) 024003 [arXiv:gr-qc/0402005].

[62] D. Mattingly, T. Jacobson and S. Liberati, Phys. Rev. D 67 (2003) 124012 [arXiv:hep$\mathrm{ph} / 0211466]$.

[63] T. J. Konopka and S. A. Major, New J. Phys. 4, 57 (2002) [arXiv:hep-ph/0201184].

[64] J. M. Carmona and J. L. Cortes, Phys. Lett. B 494 (2000) 75 [arXiv:hep-ph/0007057].

[65] E. Armengaud, G. Sigl, T. Beau and F. Miniati, Astropart. Phys. 28 (2007) 463 [arXiv:astro$\mathrm{ph} / 0603675]$.

[66] J. N. Bahcall and E. Waxman, Phys. Lett. B 556 (2003) 1 [arXiv:hep-ph/0206217].

[67] T. P. A. Collaboration, Phys. Rev. D 79 (2009) 102001 [arXiv:0903.3385 [astro-ph.HE]].

[68] E. Waxman and J. N. Bahcall, Phys. Rev. D 59 (1999) 023002 [arXiv:hep-ph/9807282].

[69] J. N. Bahcall and E. Waxman, Phys. Rev. D 64 (2001) 023002 [arXiv:hep-ph/9902383].

[70] G. Amelino-Camelia, M. Arzano, Y. J. Ng, T. Piran and H. Van Dam, JCAP 0402 (2004) 009 [arXiv:hep-ph/0307027].

[71] D. Fargion, B. Mele and A. Salis, Astrophys. J. 517 (1999) 725 [arXiv:astro-ph/9710029].

[72] R. Aloisio, V. Berezinsky, P. Blasi, A. Gazizov, S. Grigorieva and B. Hnatyk, Astropart. Phys. 27 (2007) 76 [arXiv:astro-ph/0608219].

[73] P. F. Harrison, D. H. Perkins and W. G. Scott, Phys. Lett. B 530 (2002) 167 [arXiv:hep$\mathrm{ph} / 0202074]$. 